\title{
Stenocaris pontica Chappuis \& Serban, 1953 und Stenocaropsis similis Cottarelli \& Venanzetti, 1989 (Copepoda, Harpacticoida) aus dem Mittelmeer mit einer Revision der Gattungen Stenocaropsis Apostolov, 1982 und Stenocaris Sars, 1909
}

\author{
Helmut Kunz \\ Im Allmet 8, 66132 Saarbrücken, Deutschland
}

Keywords: Taxonomy, Stenocaris, Stenocaropsis, Copepoda, Harpacticoida, Mediterranean

\begin{abstract}
Stenocaris pontica Chappuis \& Serban is for the first time recorded from the Mediterranean Sea (Croatic coast) and described. Stenocaropsis similis Cottarelli \& Venanzetti, also recorded from the Croatic coast, was compared with type material of Stenocaropsis pristina (Wells). This led to a new separation of the two species. Stenocaropsis valkanovi (Marinov) is regarded as species inquirenda. The genera Stenocaropsis Apostolov and Stenocaris Sars are revised.
\end{abstract}

\section{Zusammenfassung}

Stenocaris pontica Chappuis \& Serban wird erstmalig aus dem Mittelmeer (kroatische Küste) nachgewiesen und mit den charakteristischen Merkmalen dargestellt. Die ebenfalls an der kroatischen Küste gefundene Art Stenocaropsis similis Cottarelli \& Venanzetti wurde mit Typenmaterial von Stenocaropsis pristina (Wells) verglichen, was zu einer neuen Abgrenzung der Arten führte. Stenocaropsis valkanovi (Marinov) wird als species inquirenda betrachtet. Es wird eine Revision der Gattungen Stenocaropsis Apostolov und Stenocaris Sars durchgeführt.

\section{Einleitung}

Die ständig ansteigende Zahl bekannt werdender mariner Harpacticoiden-Arten erfordert bei der Identifizierung besondere Sorgfalt. Zur Charakterisierung der Arten müssen oft Merkmale herangezogen werden, die bisher wenig Beachtung fanden. Schwierigkeiten ergeben sich häufig, wenn ältere Arbeiten benützt werden müssen, in welchen wesentliche Merkmale unvollkommen dargestellt sind. Bei Stenocaris pontica Chappuis \& Serban, die bisher nur aus dem Schwarzen Meer bekannt war, hat mich die weite Entfernung meines Fundortes (Adriatisches Meer) zu einer Darstellung der wesentlichen Merkmale veranlaßt.

Stenocaropsis similis Cottarelli \& Venanzetti, bisher nur von Sardinien bekannt, wurde ebenfalls an der adriatischen Küste wiedergefunden. Um die Ergebnisse meiner Identifizierung sicherzustellen, wurde Typenmaterial der nahe verwandten Stenocaropsis pristina (Wells, 1968) (bisher nur von Cornwall, England bekannt) herangezogen.

\section{Material und Methoden}

Das meiner Arbeit zugrunde liegende Material habe ich zusammen mit meiner Frau 1985 und 1986 an zwei Stränden der dalmatinischen Küste (Adriatisches Meer) durch Auswaschen aus dem Küstensand gewonnen. Zusätzliches Material erhielt ich vom "British Museum of Natural History, London".

Die Konservierung der Tiere erfolgte in einem Gemisch aus 80 Vol\% auf 3,5\% verdünnter wässriger Formaldehydlösung und $20 \mathrm{Vol} \%$ Glyzerin. Die zerlegten Tiere wurden auf Objektträgern in Polyvinyllactophenol eingebettet. Zum Anfärben des Chitins wurde Orange $\mathbf{G}$ zugesetzt.

Folgende Abkürzungen wurden im Text der Arbeit verwendet: Anop Analoperculum, A1 erste Antenne, A2 zweite Antenne, Enp Endopodit, Exp Exopodit, Fu Furka, Gf Genitalfeld, Md Mandibel, Mdp Mandibularpalpus, Mxl erste Maxille, Mx2 zweite Maxille, Mxp Maxilliped, P1-P6 erstes bis sechstes Bein, R Rostrum.

Das bearbeitete Material wurde dem Zoologischen Museum der Universität Hamburg zur Aufbewahrung übergeben. Die dort registrierten Tiere sind unter ZMH Nr. aufgeführt. 
Ergebnisse

Fam. Cylindropsyllidae Sars, 1909, emend. Lang, 1948

Subfam. Cylindropsyllinae Lang, 1948

Stenocaris Sars, 1909

Stenocaris pontica Chappuis \& Serban, 1953 (Fig. 1-3)

Syn. - Stenocaris pygmaea Noodt, 1955a (nach Marinov, 1971).

Fundort und Material. - Mittelmeer: Kroatien, Insel Korčula, Stadt, beim Hotel Bon Repos, Grobsand mit Kies, 5.5.1985: einige $९$, $\sigma^{\circ} \sigma^{\circ}$ und Copepodide, ZMH Nr. K-34919 (26a-b, 27a, 51).

Bemerkungen. - S. pontica ist bisher von verschiedenen Orten am Schwarzen Meer und - als $S$. pygmaea - von der europäischen Atlantikküste gemeldet worden. Noodts Beschreibung weicht von den Beschreibungen aus dem Schwarzen Meer (Chappuis \& Serban, 1953; Marinov, 1971; Apostolov, 1973a) ab: Der Enp von P2 $\odot$ ist 2gliedrig dargestellt, während er in den Beschreibungen aus dem Schwarzen Meer immer 1gliedrig erscheint, wie auch bei meinen Tieren (Fig. 2B-C). Marinov (1971) und Apostolov (1972) betrachten pygmaea als synonym mit pontica. Eine Nachprüfung von Noodts Befund anhand von Tieren aus dem Atlantikbereich sollte aber erfolgen. Einige beigefügte Abbildungen (Fig. 3B-D, F) sollen die merkwürdigen Ausbildungen von P2 $\sigma^{\circ}$, P3 $\sigma^{\circ}$ und P6 $\sigma^{\circ}$ genauer als bisher dargestellt zeigen.

Geographische Verbreitung. - Schwarzes Meer: Rumänien (Chappuis \& Serban, 1953), Bulgarien (Marinov, 1971; Apostolov, 1972, 1973a; Marinov \& Apostolov, 1983), Ukraine (Vorobjeva, 1984), Krim (Griga, 1964). Mittelmeer: Kroatien (diese Arbeit). Europäische Atlantikküste: Frankreich: Golf von Gascogne (Noodt, 1955a, b; DelamareDeboutteville, 1954; Delamare-Deboutteville \& al., 1954; Renaud-Debyser, 1963; Renaud-Debyser \& Salvat, 1963), Bretagne (Bodin, 1988b), England/ Devon (Wells, 1963), Schottland (Hockin, 1984 und weitere Arbeiten).
Stenocaropsis Apostolov, 1982

Syn. - Stenocaris Sars, 1909 part.

Bemerkung. - Apostolov (1982) hat pristina Wells, 1968 und valkanovi Marinov, 1973 von Stenocaris abgetrennt und in die von ihm neu begründete Gattung Stenocaropsis überführt. Mit beiden Arten ist Stenocaropsis similis Cottarelli \& Venanzetti, 1989 nahe verwandt. Bei einem dieser Gruppe von Arten zugehörigen Männchen von der dalmatinischen Küste fand ich Merkmale, die für verschiedene der vorstehend genannten Arten spezifisch sein sollten. Zur Klärung der Artzugehörigkeit meines Tieres habe ich Typenmaterial von S. pristina (Wells) herangezogen.

Stenocaropsis pristina (Wells, 1968)

(Fig. 4-5)

Fundort und Material. - Scilly Inseln, Cornwall, leg. Wells; British Museum of Natural History, London No. 1967.11.1414: Holotypus $\%$, Allotypus $\sigma$, Paratypen 1 \%, $1 \%$.

Beschreibung. - Weibchen: An dem 1gliedrigen Exp A2 war beim Paratypus außer einer langen Terminalborste noch eine kurze, sehr feine Borste $\mathrm{zu}$ erkennen. Diese war beim Holotypus durch die lange Borste verdeckt und wohl daher von Wells nicht beschrieben worden.

Enp P2 (Fig. 4B): Die Innenrandborste sitzt am distalen Ende des Grundglieds - bei Wells ist sie irrtümlich proximal am Endglied gezeichnet.

Enp P4 (Fig. 4D): Das Endglied trägt apikal eine lange und eine kurze Borste; bei Wells ist hier nur eine Borste, die wohl der längeren entspricht, dargestellt.

P1 und P3 (Fig. 4C) sowie die Exp P2-P4 entsprechen der Beschreibung von Wells.

Daraus ergibt sich folgende - von Wells' Beschreibung abweichende - Borstenformel:

$\begin{array}{lcc} & \text { Exp } & \text { Enp } \\ \text { P2 } & 0.0 .022 & 1.011 \\ \text { P3 } & 0.0 .122 & 0.111 \\ \text { P4 } & 0.0 .122 & 0.011\end{array}$

Ischwach ausgebildete Borste 

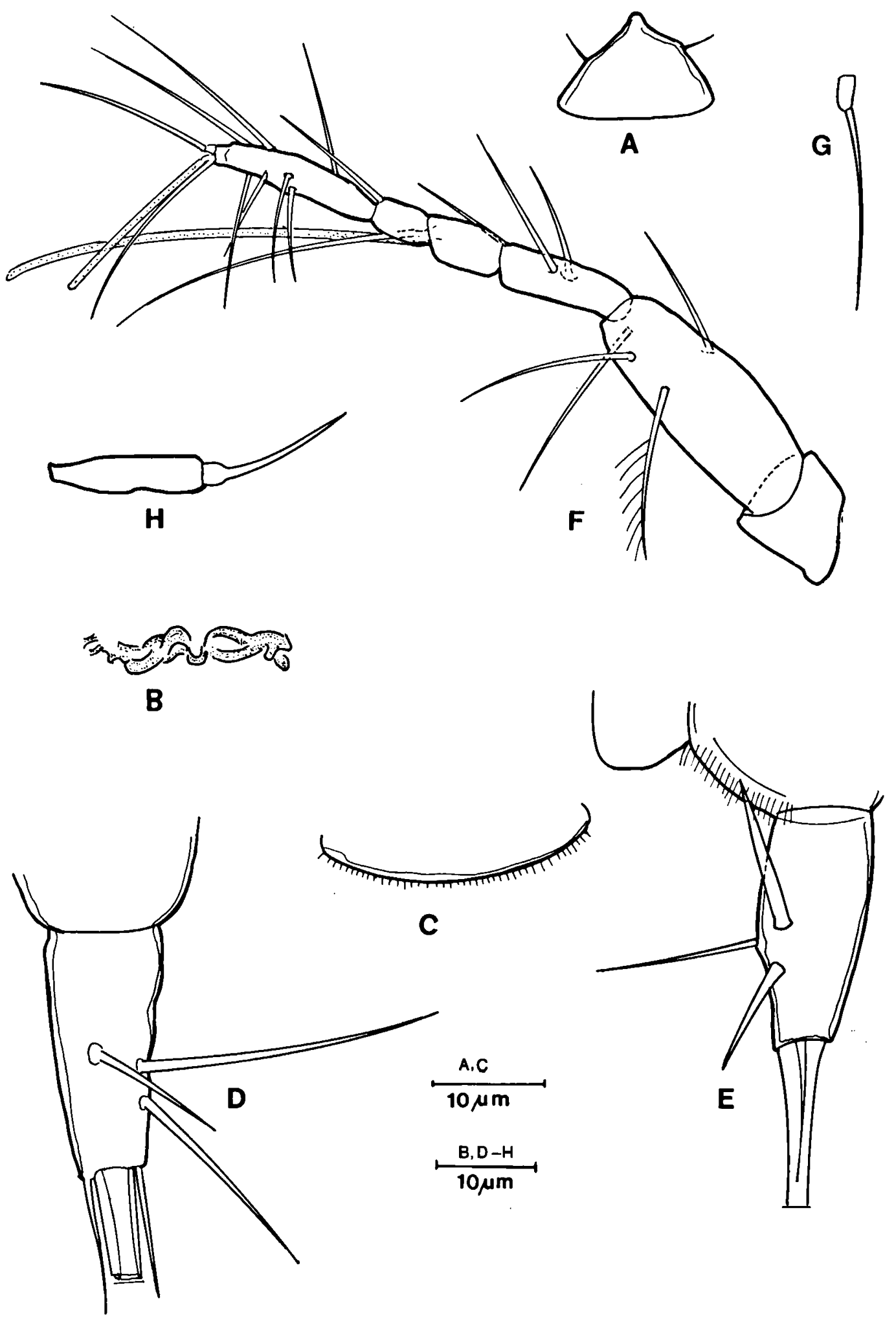

Fig. 1. Stenocaris pontica Chappuis \& Serban, Q: A, R; B, Gf; C, Anop; D, Fu, dorsal; E, Fu, lateral; F, A1; G, Exp von A2; H, Mxp. 
118

H. Kunz - Stenocaris pontica \& Stenocaropsis similis

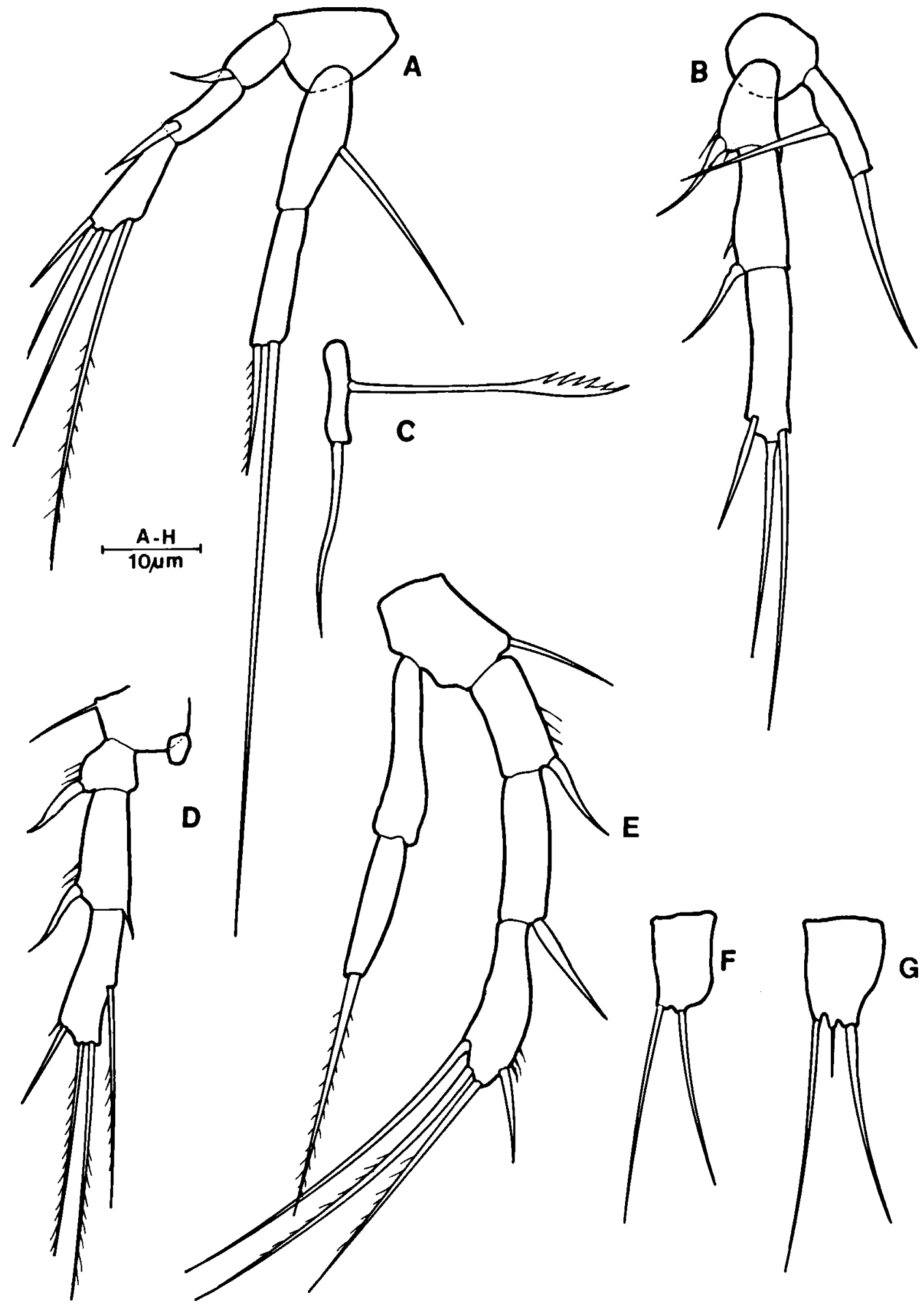

Fig. 2. Stenocaris pontica Chappuis \& Serban, \&: A, P1; B, P2; C, Enp von P2; D, P3; E, P4; F, P5; G, P5, abnorm.

Downloaded from Brill.comఠ4/26/2023 $04: 58: 42 \mathrm{AM}$

via free access 


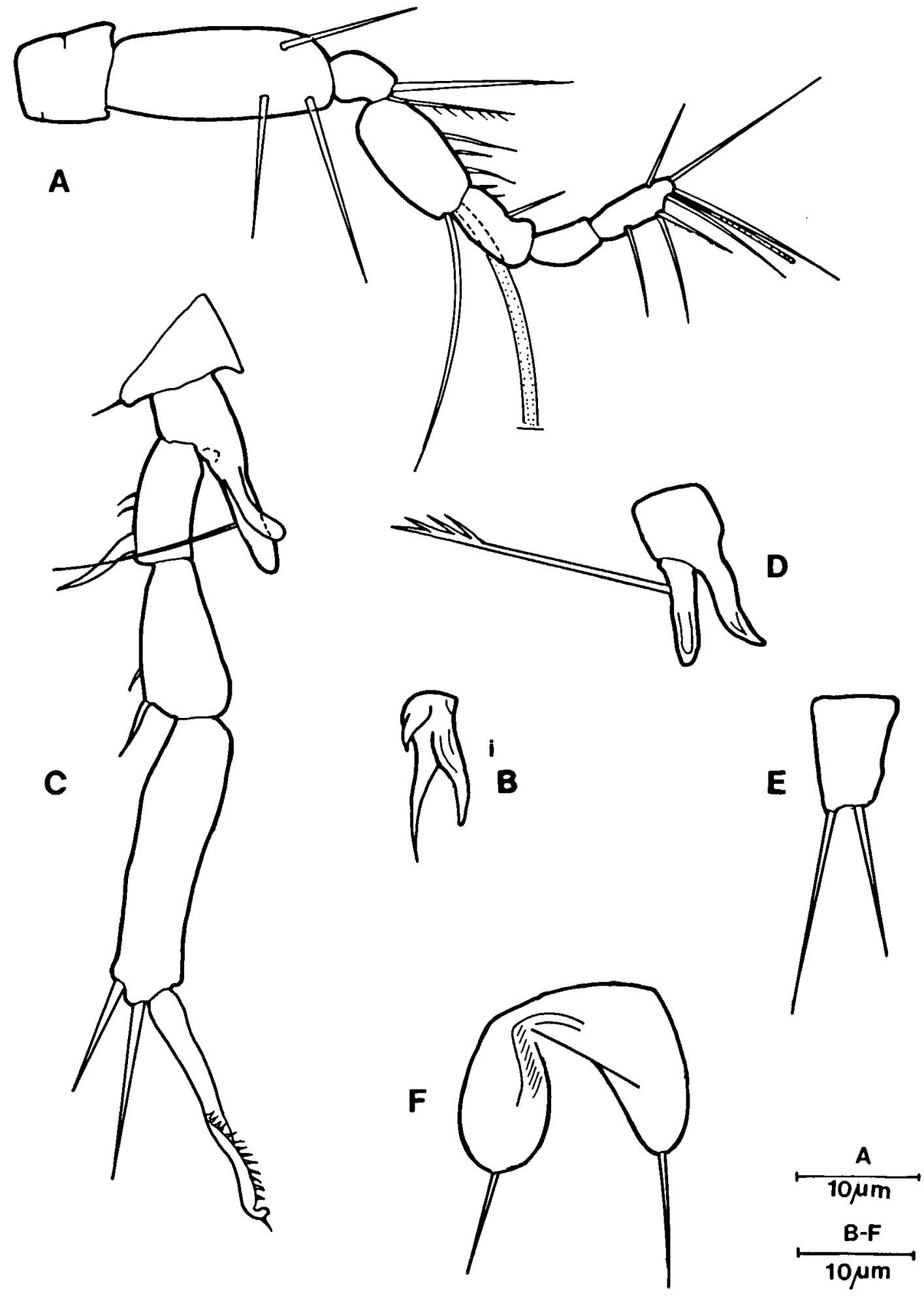

Fig. 3. Stenocaris pontica Chappuis \& Serban, $\sigma:$ A, A1; B, Enp von P3, i = Innenseite; C, P2; D, Enp von P2, lateral; E, P5; F, P6+ P6. 

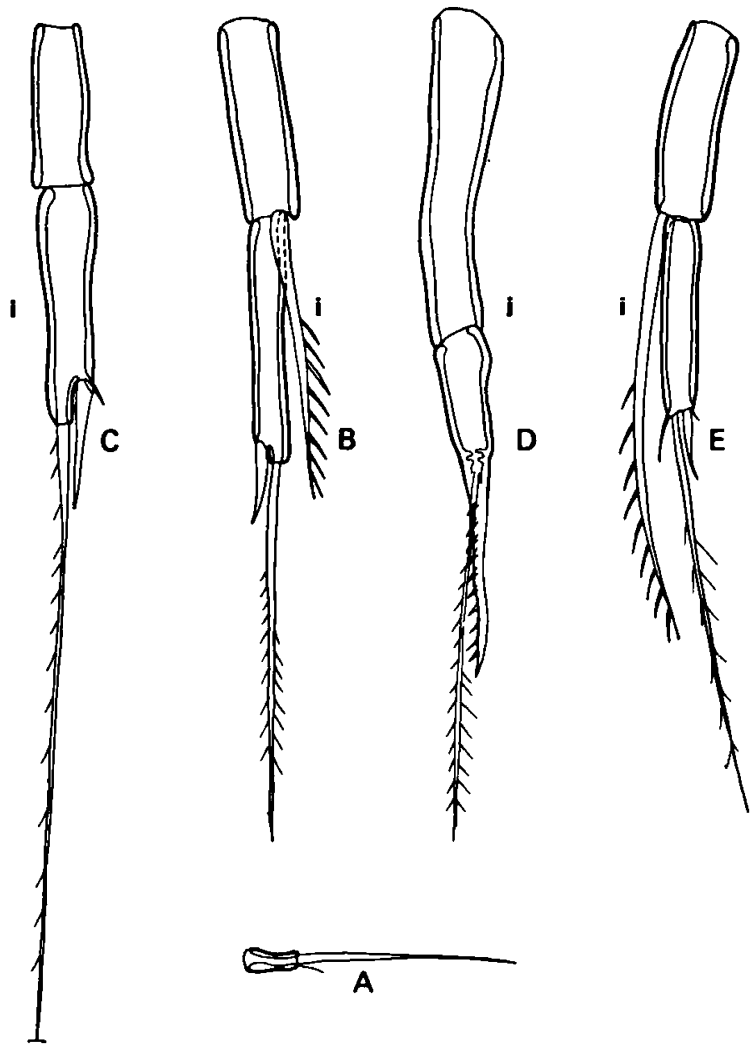

Fig. 4. Stenocaropsis pristina (Wells): A, $\propto$, Exp von A2; B, ८, Enp von $\mathrm{P} 2 ; \mathrm{C}, \%$, Enp von $\mathrm{P3}$; D, $\odot$, Enp von $\mathrm{P} 4$; E, $\sigma$, Enp von $\mathbf{P} 2, \mathbf{i}=$ Innenseite.

Männchen: A1 7gliedrig, entsprechend Fig. 14A bei Wells - im Text hat Wells 8 Glieder angegeben. Exp A2 (Fig. 4A) wie beim Weibchen.

Am Grundglied des Exp P1 sitzt eine Außenrandborste (Fig. 5A), die der des Weibchens entspricht.

Der Enp P3 (Fig. 5B) war beim Allotypus und dem von mir untersuchten Paratypus gleich gebaut und entsprach der Darstellung von Wells.

Enp P4 (Fig. 5C) entsprach dem des Weibchens.

Geographische Verbreitung. - England, Cornwall (Wells, 1968).

Stenocaropsis similis Cottarelli \& Venanzetti, 1989 (Fig. 6-9)

Fundort und Material. - Mittelmeer: Adriatisches Meer, Dalmatien, Insel Mljet, Soline, Grobsand am Ufer, leg. Kunz, 4.5.1986: 1 o, ZMH Nr. K-34940 (28a-f).
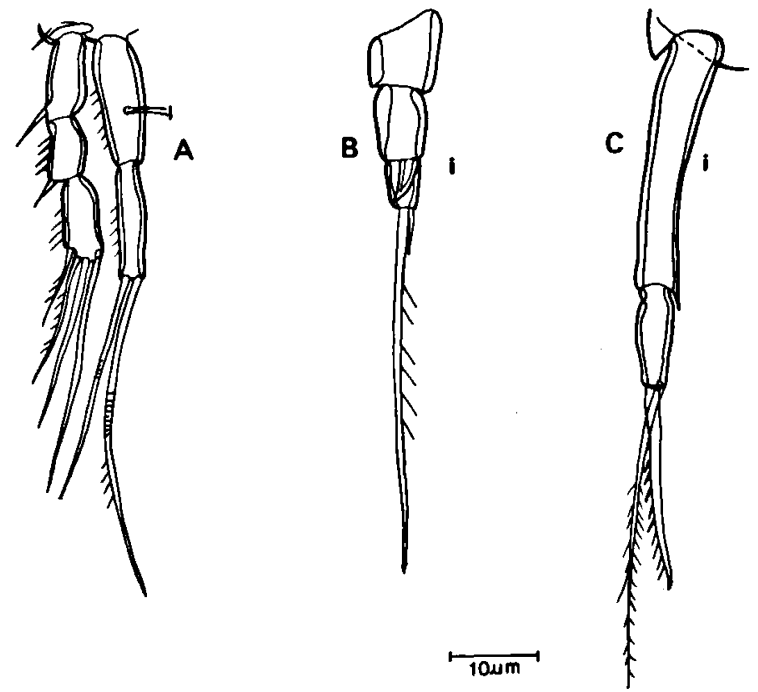

Fig. 5. Stenocaropsis pristina (Wells), o : A, P1; B, Enp von P3; C, Enp von P4, $\mathrm{i}=$ Innenseite.

Beschreibung. - Ich führe hier nur die wesentlichen bei der Unterscheidung von $S$. similis, $S$. pristina und $S$. valkanovi zu beachtenden Merkmale auf. Männchen:

Fu (Fig. 6A-B): an der Innenseite ein stumpfes Dörnchen.

A2 (Fig. 7A-B): Exp 1 gliedrig, apikal mit einer langen und einer kurzen Borste.

Mxp (Fig. 7F): Basis ohne Borste.

P1 (Fig. 8B): Am Grundglied des Exp anstelle einer Borste ein kleiner Knopf.

P2 (Fig. 8C): Grundglied des Exp mit stumpfem Vorsprung. Enp: Grundglied außen konkav, Endglied mit 3 Apikalborsten, die innere am längsten und befiedert, die mittlere kurz, ohne Fiedern, die äußere sehr kurz, haarartig.

P3 (Fig. 9A-B): Enp 3gliedrig. Mittleres Glied kaum länger als breit, mit 2 Apophysen, die äußere bis zum distalen Ende des Endglieds reichend, terminal mit kräftigen Fiedern (Fig. 9B), die innere bis zur Mitte des Endglieds reichend, ohne Fiedern. Endglied etwa 3 mal so lang wie breit, apikal mit 2 Borsten, die äußere etwa so lang wie das Glied, befiedert, die innere wesentlich kürzer, ohne Fiedern.

P4 (Fig. 9C): Enp mit 2 Apikalborsten am Endglied.

Geographische Verbreitung. - Italien: Toskanische 

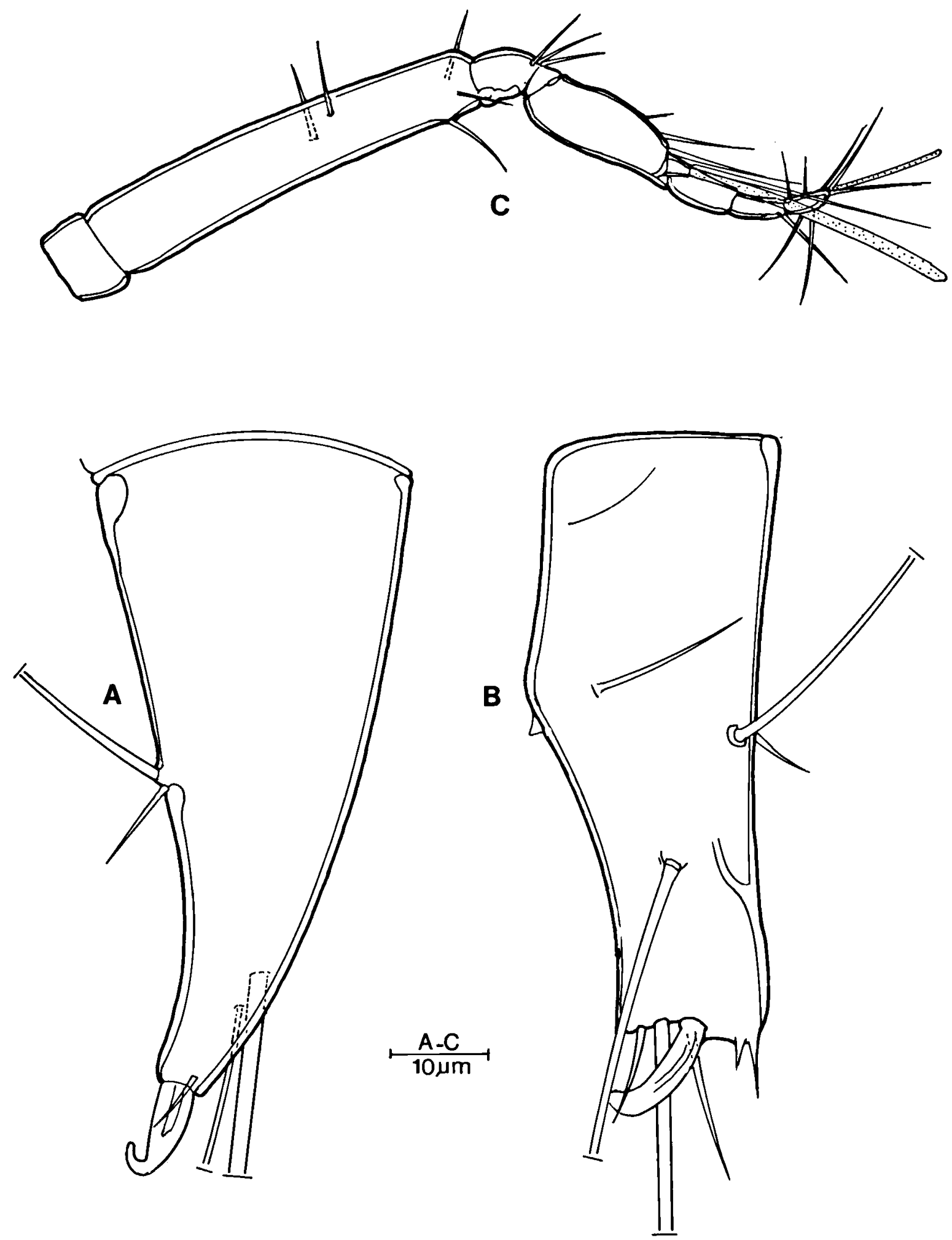

Fig. 6. Stenocaropsis similis Cottarelli \& Venanzetti, ơ: A, Fu, lateral; B, Fu, dorsal; C, A1. 


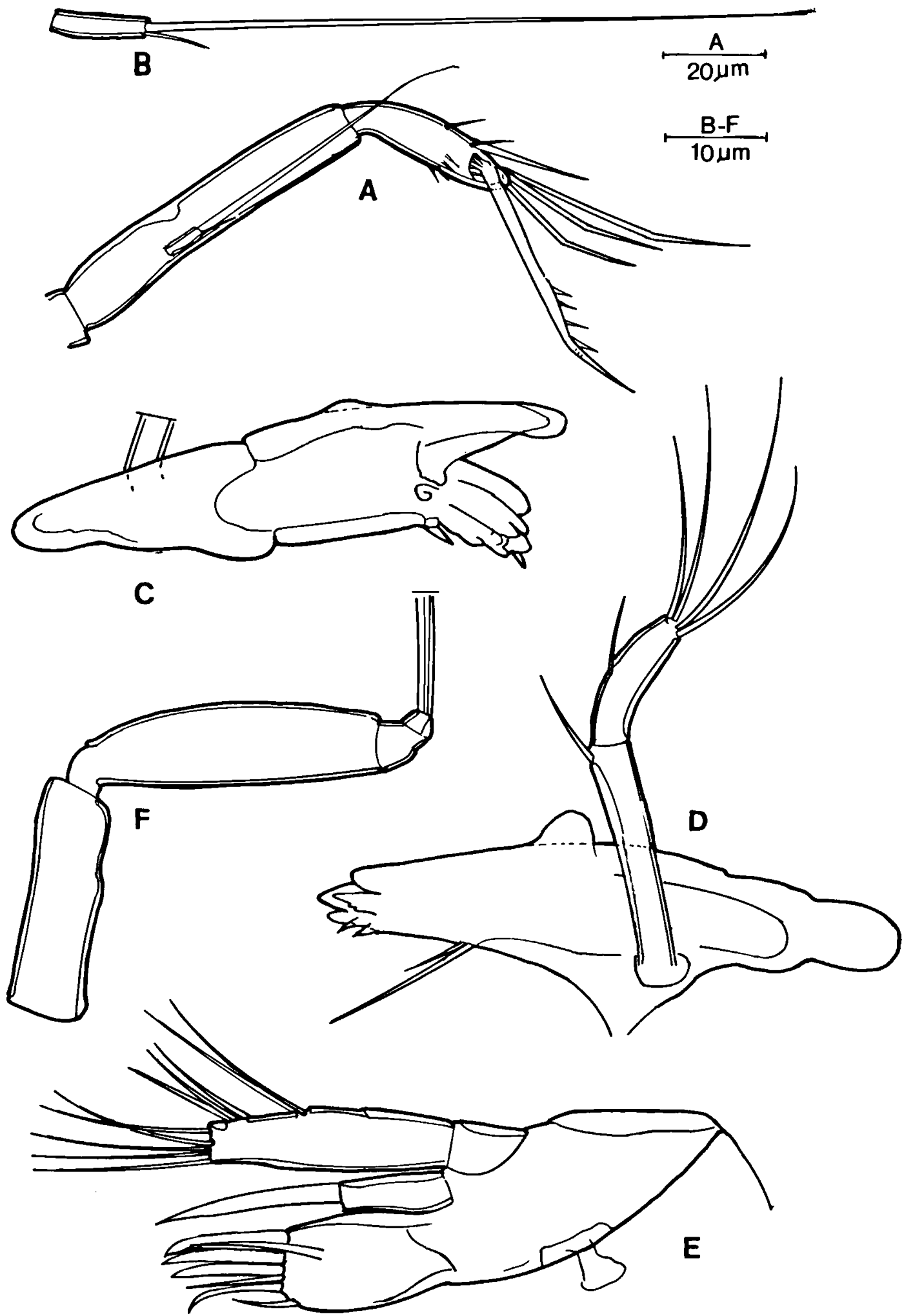

Fig. 7. Stenocaropsis similis Cottarelli \& Venanzetti, ơ: A, A2; B, Exp von A2; C, Kaulade der Md; D, Md; E, Mx1; F, Mxp. 


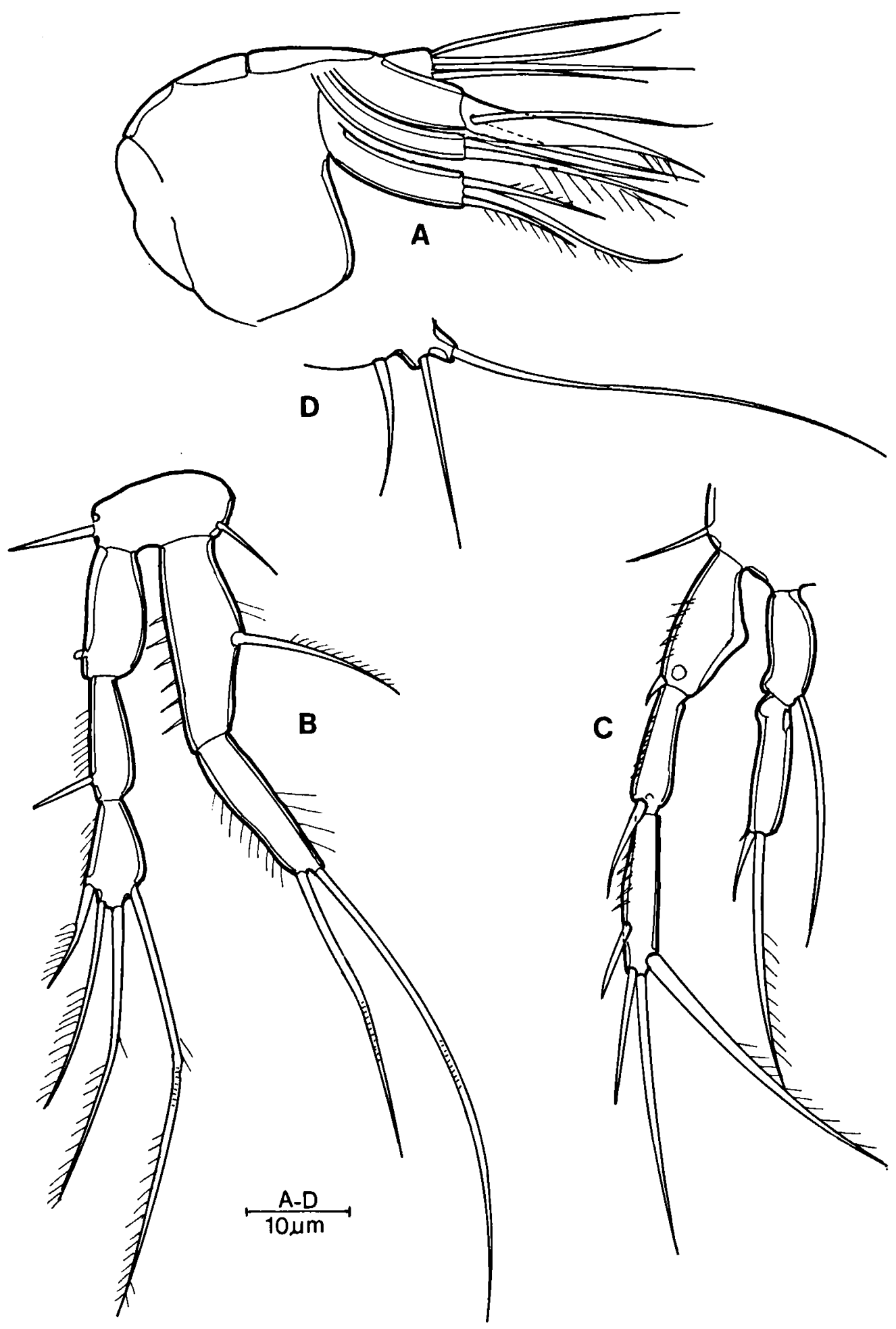

Fig. 8. Stenocaropsis similis Cottarelli \& Venanzetti, ơ: A, Mx2; B, P1; C, P2; D, P6. 
Inseln (Cottarelli \& Venanzetti, 1989), Kroatien, Insel Mljet (diese Arbeit).

Diskussion zur Art. - Stenocaropsis pristina, similis und valkanovi sind nahe miteinander verwandt. Die Weibchen von pristina und similis haben die gleiche Borstenformel und sind nicht zu unterscheiden. Bei den Männchen bestehen Unterschiede: Die Außenrandborste am Grundglied des Exp P1 ist bei pristina normal (Fig. 5A), bei similis hingegen zu einem kleinen Knopf reduziert (Fig. 8B). Cottarelli \& Venanzetti haben bei der Beschreibung des Männchens von similis keine Angaben über Unterschiede im Bau des P1 ơ gegenüber dem des Weibchens gemacht d.h., daß die von mir festgestellte Veränderung der Außenrandborste am Grundglied des Exp P1 O’ von Cottarelli \& Venanzetti nicht beobachtet wurde. Am Mittelglied des Enp P3 $\sigma^{\prime}$ konnte ich bei pristina nur eine Apophyse beobachten (Fig. 5B) gegenüber 2 bei similis (Fig. 9A-B).

$S$. valkanovi und $S$. similis sind wahrscheinlich synonym. Die Borstenformeln der Weibchen sind gleich. Unterschiede: Der Exp A2 soll entsprechend der Beschreibung Marinovs (1973) lediglich durch eine Borste repräsentiert sein. Dies ist unwahrscheinlich, denn die beiden anderen StenocaropsisArten besitzen einen 1gliedrigen Exp A2. Außerdem ist bei valkanovi am Mittelglied des Enp P3 O* nur eine Apophyse gezeichnet an Stelle von 2 bei similis; die kürzere kann jedoch leicht übersehen werden. Das gleiche gilt für die Außenrandborste am Grundglied des Exp P1 $\sigma^{\circ}$, die bei similis zu einem kleinen Knopf reduziert ist (Fig. 8B) und bei valkanovi (Marinov, 1973, Fig. 4) vollständig fehlen soll. $S$. valkanovi ist als species inquirenda $\mathrm{zu}$ betrachten.

Geographische Verbreitung von S. valkanovi (Marinov, 1973). - Schwarzes Meer: Krim (Griga, 1964), Bulgarien (Apostolov, 1970; Marinov, 1973).

\section{Revision der Gattungen Stenocaropsis Apostolov, 1982 und Stenocaris Sars, 1909}

Apostolov (1982) hat Stenocaris pristina Wells, 1968 und Stenocaris valkanovi Marinov, 1973 von der Gattung Stenocaris abgetrennt und in die von ihm neu begründete Gattung Stenocaropsis überführt. Zur Begründung zitiert er Marinov (1973: 312): "les deux espèces sont isolées des autres formes du genre [Stenocaris] qui se caractérise par un endopodite uniarticulé au P2, P4, tandis que l'endopodite de ces deux espèces est biarticulé'. Zweifellos haben Marinov und Apostolov bei dieser Festellung den P3 statt des P4 gemeint. Bereits Wells (1968: 419) hat die Sonderstellung von pristina hervorgehoben: "This is undoubtedly the most primitive species of Stenocaris yet discovered" und bezieht sich dabei auf die 2gliedrigen Enp von P2 und P3 sowie eine reichere Beborstung von P2-P4 bei pristina gegenüber anderen Stenocaris-Arten.

Es müssen aber noch zwei weitere von Apostolov nicht berücksichtigte Arten zu Stenocaropsis gestellt werden, nämlich die von Becker (1979) aus der iberischen Tiefsee beschriebenen Arten Stenocaris abyssalis und Stenocaris profundus, die ebenfalls 2gliedrige Enp P2 \& aufweisen. Huys (1988: 270) hat bereits auf diese Tatsache hingewiesen, ohne zur Frage der Gattungszugehörigkeit der genannten Arten Stellung zu nehmen.

\section{Gattungsdiagnose von Stenocaropsis Apostolov, 1982}

Weibchen: Körper zylindrisch. Rostrum an der Basis abgesetzt. Fu länger als breit, Terminalborsten normal. A1 5-7gliedrig. A2 mit Allobasis, Enp mit 5-8 Borsten, Exp 1gliedrig mit 2 Borsten. Mdp 1-2gliedrig. Mx1 mit 2(1?) Borsten an der Coxa. Mx2 mit 2 Enditen. Mxp: Basis mit oder ohne Borste an der distalen inneren Ecke; erstes Glied des Enp glatt; Endglied mit Klaue, mit oder ohne kurzer Begleitborste. P1-P4 mit 3gliedrigen Exp und 2gliedrigen Enp. Endglieder der Exp mit 4,4,5,5 Borsten, Endglieder der Enp mit 2,3,3,2 Borsten. P5 1gliedrig mit 5-8 Borsten.

Männchen: Sexualdimorphismen am Exp P2 und Enp P3. P5 1gliedrig mit 5-6 Borsten.

Fünf Arten:

Stenocaropsis pristina (Wells, 1968)

Syn.: Stenocaris pristina Wells, 1968, \&, O"; Bodin, 1970; Itô, 1972; Wells, 1986. 


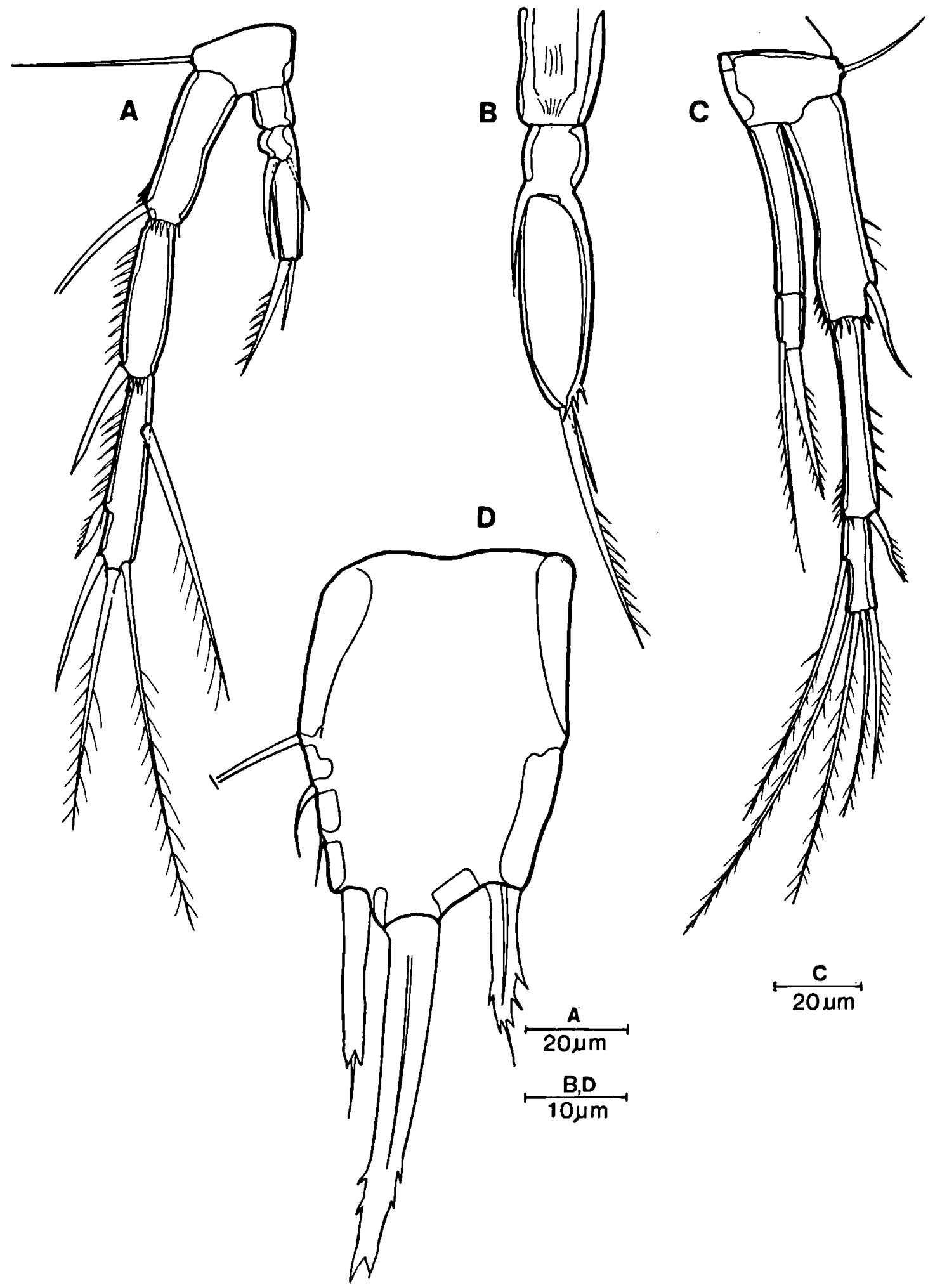

Fig. 9. Stenocaropsis similis Cottarelli \& Venanzetti, ơ: A, P3; B, Enp von P3; C, P4; D, P5. 
Stenocaropsis profundus (Becker, 1979)

Syn.: Stenocaris profundus Becker, 1979, \&; Bodin, 1979, 1988; Schriever, 1979; Willems, 1981.

Stenocaropsis abyssalis (Becker, 1979)

Syn.: Stenocaris abyssalis Becker, 1979, O"; Bodin, 1988; Schriever, 1979; Willems, 1981.

Stenocaropsis valkanovi (Marinov, 1973)

Syn.: Stenocaris valkanovi Marinov, 1973, \&, ơ ; Bodin, 1976, 1979; Wells, 1986; Arenopontia sp. Griga, 1964; Apostolov, 1970, 1971; Stenocaris pristina Apostolov, 1973b.

Stenocaropsis similis Cottarelli \& Venanzetti, 1989.

\section{Bestimmungsschlüssel zu Stenocaropsis}

Weibchen:

1. A1 5gliedrig, Ästhetask am 3. Glied; P5 mit 5 Borsten ... .......................... profundus

- A1 6gliedrig, Ästhetask am 4. Glied, P5 mit 8 Borsten ... 2

2. Exp A2 1gliedrig mit 2 Borsten ........ pristina, similis

- Exp A2 nur durch eine Borste repräsentiert .... valkanovi Männchen:

1. Endglied Exp P2 stark einwärts gekrümmt, mit 2 Borsten; P5 mit 5 Borsten ................... abyssalis

- Endglied Exp P2 gegenüber dem des Weibchens nicht modifiziert, gerade, mit 4 Borsten am Endglied ....... 2

2. Grundglied Exp P1 mit Außenrandborste ........ pristina

- Am Grundglied Exp P1 anstelle der Außenrandborste ein Knopf $\ldots \ldots \ldots \ldots \ldots \ldots \ldots \ldots \ldots \ldots \ldots \ldots$

3. Exp A2 1gliedrig mit 2 Borsten, mittleres Glied Enp P3 mit 2 Borsten bzw. Apophysen ............... similis

- Exp A2 nur durch eine Borste repräsentiert, mittleres Glied Enp P3 mit einer Apophyse ............ valkanovi

\section{Gattungsdiagnose von Stenocaris Sars, 1909}

Weibchen: Körperform zylindrisch. $R$ an der Basis abgesetzt. Fu quadratisch oder länger als breit, Terminalborste normal oder dornförmig. Al 6-7gliedrig, Ästhetask am vierten Glied. Exp A2 1gliedrig mit 1-2 Borsten. Mdp 1-2gliedrig. Mx1 mit 2 Borsten an der Coxa. Mxp mit oder ohne Borste an der Basis, erstes Glied des Enp glatt oder mit Borstenreihe, Endglied mit oder ohne kurze Begleitborste. Exp P1-P4 3gliedrig, Enp P1 und P4 2gliedrig, Enp P2 und P3 1gliedrig. Endglieder Exp P1-P4 mit 3-4, 3-4, 4-5, 3-5 Borsten; Endglieder Enp P1-P4 mit 2-3, 2, 1-2, 1-2 Borsten.
P5 mit 2-8 Borsten oder spitzen Vorsprüngen. Männchen: Modifikationen gegenüber dem Weibchen an Fu, A1, Enp P2 und P3. P5 mit 2-7 Borsten oder spitzen Vorsprüngen.

Neun Arten:

Stenocaris minor (T. Scott, 1892)

Syn.: Cylindropsyllus minor T. Scott, 1892, ९, o'.

Stenocaris gracilis Sars, 1909, \&, o . Stenocaris arenicola Wilson, 1932, ơ . Stenocaris minuta Nicholls, 1935, ९, $0^{\circ}$. Stenocaris kliei Kunz, 1936, \&, o“.

Stenocaris pontica Chappuis \& Serban, 1953, \&, $\sigma^{\circ}$.

Syn.: Stenocaris pygmaea Noodt, 1955a, Q; Bodin, 1967, 1976; Hockin, 1984 u.a.; Itô, 1972; Marinov, 1971; Renaud-Debyser, 1963; RenaudDebyser \& Salvat, 1963; Wells, 1963.

Stenocaris intermedia Itô, 1972, Q, O*. Stenocaris kerguelenensis Bodiou, 1977, ९, ० . Stenocaris baltica Arlt, 1983, O".

$S$. baltica ist mit minuta nahe verwandt (oder synonym ?). Bei dem in der Beschreibung als P5 bezeichneten Bein handelt es sich um P6.

Die Gattung Stenocaris ist nicht einheitlich zusammengesetzt. Sie umfaßt zwei deutlich voneinander getrennte Gruppen:

(1) Die minor-Gruppe: Exp A2 gut entwickelt mit 2 Borsten; P5 \& mit 7-8 Borsten oder spitzen Vorsprüngen; P5 o mit 5-6 Borsten oder spitzen Vorsprüngen; Endglied Exp P2 mit 4 Borsten, Endglied Exp P3 mit 5 Borsten. Hierher gehören minor, gracilis, arenicola, kliei, intermedia und kerguelenensis.

(2) Die minuta-Gruppe: Exp A2 schwach entwikkelt, mit nur einer Borste; P5 $\%$ mit 2-3 Borsten; P5 o mit 2 Borsten; Endglied Exp P2 mit 3 Borsten, Endglied Exp P3 mit 4 Borsten. Hierher gehören minuta, pontica und baltica.

\section{Bestimmungsschlüssel zu Stenocaris}

\section{Weibchen:}

1. Endglied Exp P2 mit 3 Borsten, Endglied Exp P3 mit 4 Borsten, P5 mit 2-3 Borsten (minuta-Gruppe) ......... 2 
- Endglied Exp P2 mit 4 Borsten, Endglied Exp P3 mit 5 Borsten, P5 mit 7-8 Borsten bzw. entsprechenden Vorsprüngen

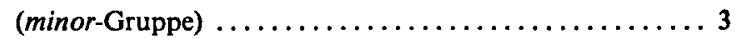

2. Enp P3 mit 2 Borsten, P5 mit 3 Borsten ....... minuta

- Enp P3 klein, ohne Borsten; P5 mit 2 Borsten ... pontica

3. Mittlere Terminalborste der Fu flaschenförmig (Bodiou, 1977, Fig. 1G-H) ................. kerguelenensis

- Mittlere Terminalborste der Fu dornförmig ......... 4

- Mittlere Terminalborste der Fu normal ........... 5

4. Dorn am distalen Ende der Fu etwas länger als die $\mathrm{Fu} . .$. .................................. minor

- Dorn am distalen Ende der Fu weniger als halb so lang wie die Fu .............................. intermedia

5. Fu doppelt so lang wie breit, Oberseite ohne Dorn, P5 mit 7 Borsten und einem fingerförmigen Vorsprung .... gracilis

- Fu wenig länger als breit, Oberseite mit Dorn, P5 mit 6 Borsten und einem fingerförmigen Vorsprung ........kliei Männchen:

1. Exp A2 gut entwickelt, mit 2 Borsten (minor-Gruppe) ... 2

- Exp A2 klein, mit einer Borste (minuta-Gruppe) .....6 6

2. P5 mit 7 Borsten .................... arenicola

- P5 mit 5 Borsten ................. kerguelenensis

- P5 mit 6 Borsten oder ähnlichen Vorsprüngen ....... 3

3. Fu etwas länger als breit, dorsal mit Horn, P5 mit 5 Borsten und einem fingerförmigen Vorsprung ..........kliei

- Fu etwa doppelt so lang wie breit, dorsal ohne Horn, P5 mit 6 Borsten ......................... 4

4. Endglied Exp P2 etwa 1,2 mal so lang wie das mittlere Glied

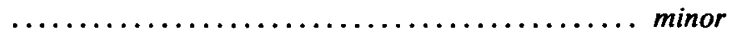

- Endglied Exp P2 etwa 3 mal so lang wie das mittlere Glied

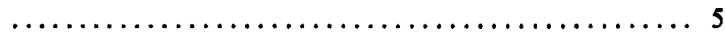

5. Endglied Enp P3 am Innenrand mit borstenähnlichem Fortsatz ............................ gracilis

- Endglied Enp P3 am Innenrand ohne borstenähnlichen Fortsatz ............................. intermedia

6. Enp P3 zangenförmig, ohne Borsten ......... pontica

- Enp P3 Igliedrig mit 2 Borsten $\ldots \ldots \ldots \ldots \ldots \ldots \ldots .7$

7. Enp P2 mit Borste am Innenrand $\ldots \ldots \ldots \ldots \ldots$ minuta

- Enp P2 ohne Borste am Innenrand ........... baltica

\section{Dank}

Ich danke meiner Frau für ihre Hilfe beim Sammeln des Materials, sowie Herrn Dr. G.F. Boxshall, Natural History Museum, London für Typenexemplare, die mir leihweise zur Verfügung gestellt wurden.

\section{Literatur}

Apostolov, A., 1970. Katalog der Harpacticoidenfauna Bulgariens. Zool. Anz., 184: 412-427.

Apostolov, A., 1971. Recherches sur la systématique et la distri- bution des Copépodes harpacticoïdes de la côte bulgare. Zool. Anz., 186: 337-447.

Apostolov, A., 1972. Catalogue des Copépodes harpacticoïdes marins de la Mer Noire. Zool. Anz., 188: 202-254.

Apostolov, A., 1973a. Sur divers Harpacticoïdes (Copépodes) de la Mer Noire. Zool. Anz., 190: 88-110.

Apostolov, A., 1973b. Notes sur les Harpacticoïdes (Crustacea, Copepoda) de la Mer Noire. Zool. Anz., 199: 175-189.

Apostolov, A., 1982. Genres et sous-genres nouveaux de la famille Diosaccidae Sars et Cylindropsyllidae Sars, Lang (Copepoda, Harpacticoidea). Acta zool. bulg., 19: 37-42.

Arlt, G., 1983. Taxonomy and ecology of some harpacticoids (Crustacea, Copepoda) in the Baltic Sea and Kattegatt. Zool. Jb. (Syst.), 110: 45-85.

Becker, K.-H. (Hrg.: W. Noodt \& G. Schriever), 1979. Eidonomie und Taxonomie abyssaler Harpacticoidea (Crustacea, Copepoda) Teil II. Paramesochridae, Cylindropsyllidae und Cletodidae. Meteor Forsch.-Ergebn., (D) 31: 1-37.

Bodin, P., 1967. Catalogue des nouveaux Copépodes Harpacticoïdes marins. Mém. Mus. nat. Hist. Nat., (A) 50 (1): 1-76.

Bodin, P., 1970 (1971). Catalogue des nouveaux Copépodes Harpacticoïdes marins. Additif no 1 . Téthys, 2 (4): 881-907.

Bodin, P., 1976. Catalogue des nouveaux Copépodes Harpacticoïdes marins. Additif № 3: 1-45 (Univ. Bretagne occidentale, Brest).

Bodin, P., 1979. Catalogue des nouveaux Copépodes Harpacticoïdes marins (nouvelle édition): 1-228 (Univ. Bretagne occidentale, Brest).

Bodin, P., 1988a. Catalogue des nouveaux Copépodes Harpacticoïdes marins (éd. 1988): 1-288 (Univ. Bretagne occidentale, Brest).

Bodin, P., 1988b. Results of ecological monitoring of three beaches polluted by the 'Amoco Cadiz' oil spill: development of meiofauna of 1978 to 1984. Mar. Ecol. Prog. Ser., 42: 105-123.

Bodiou, J.Y., 1977. Harpacticoïdes (Crustacés, Copépodes) des Iles Kerguelen. III - Description de deux formes nouvelles de la famille des Cylindropsyllidae. CNFRA (Comité national français des Recherches antarctiques), 42: 277-286.

Chappuis, P.A. \& M. Serban, 1953. Copépodes de la nappe phréatique de la plage d'Agigea près Constanza. Notes biospéol., 8: 91-102.

Cottarelli, V. \& F. Venanzetti, 1989. Cylindropsyllidae del meiobenthos di Montecristo e delle isole circumsarde (Crustacea, Copepoda, Harpacticoida). Annali Mus. Civ. Stor. nat. G. Doria, 87: 183-235.

Delamare Deboutteville, C., 1954. L'écologie du Mystacocaride Derocheilocaris remanei f. biscayensis Delamare sur les côtes du Golfe de Gascogne. Vie Milieu, 5: 310-329.

Delamare Deboutteville, C., S. Gerlach \& R. Siewing, 1954. Recherches sur la faune des eaux souterraines littorales du Golfe de Gascogne. Littoral des Landes. Vie Milieu, 5: 373407.

Griga, R.E., 1964. Copepoda from the benthonic biocoenose in the region of Eupatoria of the Black Sea. Trudy Sewastopol biol. Sta., 15: 101-117. 
Hockin, D.C., 1984. Records of symbiotic Protozoa from harpacticoid copepods of a sandy beach. Crustaceana, 46: 319-320.

Huys, R., 1988. Studies on the Cylindropsyllidae (Copepoda, Harpacticoida), 2. A revision of the genus Cylindropsyllus Brady. Zoologica Scr., 17: 253-276.

Itô, T., 1972. Descriptions and records of marine harpacticoid copepods from Hokkaido, IV. J. Fac. Sci. Hokkaido Univ., (VI, Zool.) 18: 305-336.

Kunz, H., 1936. Neue Harpacticoiden (Crustacea, Copepoda) von Helgoland. Kieler Meeresforsch., 1: 352-358.

Lang, K., 1948. Monographie der Harpacticiden: 1-1682 (Hakån Ohlson, Lund).

Marinov, T., 1971. Harpacticoids of the Bulgarian Black Sea coast. Proc. Inst. Oceanogr. Fish. Varna, 11: 43-87.

Marinov, T., 1973. Quelques Harpacticides psammophiles inconnus pour le bassin de la Mer Noire. Vie Milieu, (A) 23: 309-326.

Marinov, T. \& A. Apostolov, 1983. Zoogeography and ecology of the Black Sea harpacticoids. Proc. Inst. Fish., Varna, 20: 135-144 [in Bulg.].

Nicholls, A.G., 1935. Copepods from the interstitial fauna of a sandy beach. J. mar. biol. Ass. U.K., 20: 379-405.

Noodt, W., 1955a. Harpacticiden (Crust. Cop.) aus dem Sandstrand der französischen Biscaya-Küste. Kieler Meeresforsch., 11: 86-109.

Noodt, W., 1955b. Harpacticides (Crust. Cop.) psammiques de la côte sud-ouest de la France. Vie Milieu, 6: 151-153.

Renaud-Debyser, J., 1963. Recherches écologiques sur la faune interstitielle des sables. Bassin d'Arcachon; île de Bimini, Bahamas. Vie Milieu, Suppl. 15: 1-143.

Renaud-Debyser, J. \& B. Salvat, 1963. Eléments de prospérité des biotopes des sédiments meubles intertidaux et écologie de leurs populations en microfaune et macrofaune. Vie Milieu, 14: $463-550$.

Sars, G.O., 1903-1911. An account on the Crustacea of Norway, V. Copepoda Harpacticoida: 1-449 (Bergen Mus., Bergen).

Schriever, G., 1979. Katalog der von K.-H. Becker bearbeiteten Tiefsee-Harpacticoida (Crustacea, Copepoda) in der Sammlung des Zoologischen Museums Kiel. Mitt. zool. Mus. Univ. Kiel, 1 (1): 1-8.

Scott, T., 1892. Additions to the fauna of the Firth of Forth, IV. Ann. Rep. Fish. Board Scotl., (3) 10: 244-272.

Vorobjeva, L.V., 1984. The distribution of Copepoda (Harpacticoida) in sand of the north-west coast of the Black Sea. Biol. Morja, 3: 45-49 [in Russ.].

Wells, J.B.J., 1963. Copepoda from the littoral region of the estuary of the river Exe (Devon, England). Crustaceana, 5: 10-26.

Wells, J.B.J., 1968. New and rare Copepoda Harpacticoida from the isles of Scilly. J. nat. Hist., 2: 397-424.

Wells, J.B.J., 1986. Copepoda: marine-interstitial Harpacticoida. In: L. Botosaneanu (ed.), Stygofauna mundi: 356-381 (E.J. Brill/Dr. W. Backhuys, Leiden).

Willems, K.A., 1981. Boreopontia heipi n. g., n. sp., an interstitial harpacticoid (Copepoda) from the Southern Bight of the North Sea. Biol. Jb. Dodonaea, 49: 200-209.

Wilson, Ch.B., 1932. The copepods of the Woods Hole region Massachusetts. Bull. U.S. natn. Mus., 158: i-xix, 1-635, pls. $1-41$.

Eingegangen am 13. Dezember 1993

Revision: 6 März 1994 